\title{
Narrow-band imaging versus Lugol chromoendoscopy for esophageal squamous cell cancer screening in normal endoscopic practice: randomized controlled trial
}

\author{
Authors \\ Mélissa Gruner ${ }^{1}$, Angélique Denis ${ }^{2}$, Claude Masliah $^{3}$, Morgane Amil ${ }^{4}$, Elodie Metivier-Cesbron ${ }^{5}$, Dominique Luet ${ }^{5}$, \\ Medhi Kaasis ${ }^{6}$, Emmanuel Coron ${ }^{7}$, Marc Le Rhun ${ }^{7}$, Stéphane Lecleire ${ }^{8}$, Michel Antonietti ${ }^{8}$, Jean-Louis Legoux ${ }^{9}$, Laurent \\ Lefrou $^{9}$, Pascal Renkes ${ }^{10}$, Anne-Laure Tarreirias ${ }^{11}$, Philippe Balian ${ }^{11}$, Philippe Rey ${ }^{12}$, Bénédicte Prost ${ }^{13}$, Christophe \\ Cellier ${ }^{14}$, Gabriel Rahmi ${ }^{14}$, Elia Samaha ${ }^{14}$, Serge Fratte ${ }^{15}$, Béatrice Guerrier ${ }^{16}$, Verena Landel ${ }^{17}$, Sandrine Touzet ${ }^{2}$, \\ Thierry Ponchon1,18, 19, Mathieu Pioche1,18,19
}

\section{Institutions}

1 Gastroenterology Division, Hôpital Edouard Herriot, Hospices Civils de Lyon, Lyon, France

2 Pole de Santé Publique, Statistics and Medical Research Department, Hospices Civils de Lyon, Lyon, France

3 Gastroenterology Division, Clinique Mutualiste de l'Estuaire, Saint Nazaire, France

4 Gastroenterology Division, Centre Hospitalier Départemental Les Oudairies, La Roche sur Yon, France

5 Gastroenterology Division, Centre HospitaloUniversitaire Larrey, Angers, France

6 Gastroenterology Division, Centre Hospitalier de Cholet, Cholet, France

7 Hepatogastroenterology Department, Hotel Dieu, Nantes, France

8 Gastroenterology Division, Hôpital Charles Nicolle, Centre Hospitalo-Universitaire, Rouen, France

9 Gastroenterology Division, Hôpital La Source, Centre Hospitalier Régional, Orléans, France

10 Gastroenterology Division, Hôpital Clinique Claude Bernard, Metz, France

11 Gastroenterology Division, Hôpital Foch, Suresnes, France

12 Gastroenterology Division, Hôpital d'Instruction des Armées, Legouest, Metz, France

13 Gastroenterology Division, Centre Hospitalier Saint Joseph Saint Luc, Lyon, France

14 Gastroenterology Division, Hôpital Européen Georges Pompidou, Paris, France

15 Gastroenterology Division, Centre Hospitalier Régional, Belfort, France

16 Gastroenterology Division, Centre Hospitalier Bourg en Bresse, Bourg en Bresse, France

17 Direction Recherche Clinique et Innovations, Hospices Civils de Lyon, Lyon, France

18 Lyon 1 University Claude Bernard, Lyon, France

19 INSERM U1032, LabTAU, Lyon, France submitted 28.1.2020

accepted after revision 22.7.2020

published online 22.7.2020

Bibliography

Endoscopy 2021; 53: 674-682

DOI 10.1055/a-1224-6822

ISSN 0013-726X

(c) 2020. Thieme. All rights reserved.

Georg Thieme Verlag KG, Rüdigerstraße 14,

70469 Stuttgart, Germany

丹upplementary material

Supplementary material is available under

https://doi.org/10.1055/a-1224-6822

Scan this QR-Code for the author commentary.

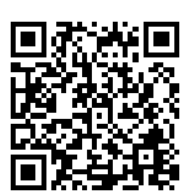

Corresponding author

Mathieu Pioche, MD, PhD, Endoscopy Unit, Digestive Disease Department, L Pavillon Edouard Herriot Hospital, 69437 Lyon Cedex, France

Fax: +33-4-72110147

mathieu.pioche@chu-lyon.fr

\section{ABSTRACT}

Background Narrow-band imaging (NBI) is as sensitive as Lugol chromoendoscopy to detect esophageal squamous cell carcinoma (SCC) but its specificity, which appears higher than that of Lugol chromoendoscopy in expert centers, remains to be established in general practice. This study aimed to prove the superiority of NBI specificity over Lugol chromoendoscopy in the detection of esophageal SCC and high grade dysplasia (HGD) in current general practice (in- 
cluding tertiary care centers, local hospitals, and private clinics).

Methods This prospective randomized multicenter trial included consecutive patients with previous or current SCC of the upper aerodigestive tract who were scheduled for gastroscopy. Patients were randomly allocated to either the Lugol or NBI group.In the Lugol group, examination with white light and Lugol chromoendoscopy were successively performed. In the NBI group, NBI examination was performed after white-light endoscopy. We compared the diagnostic characteristics of NBI and Lugol chromoendoscopy in a per-patient analysis.
Results 334 patients with history of SCC were included and analyzed (intention-to-treat) from 15 French institutions between March 2011 and December 2015. In per-patient analysis, sensitivity, specificity, positive and negative likelihood values were $100 \%, 66.0 \%, 21.2 \%$, and $100 \%$, respectively, for Lugol chromoendoscopy vs. $100 \%, 79.9 \%$, $37.5 \%$, and $100 \%$, respectively, for NBI. Specificity was greater with NBI than with Lugol $(P=0.002)$.

Conclusions As previously demonstrated in expert centers, $\mathrm{NBI}$ was more specific than Lugol in current gastroenterology practice for the detection of early SCC, but combined approaches with both $\mathrm{NBI}$ and Lugol could improve the detection of squamous neoplasia.

\section{Introduction}

Esophageal squamous cell carcinoma (SCC) is a frequent and severe disease that is associated with a high mortality rate $[1,2]$. Prognosis is related to disease stage at the time of diagnosis and is considerably better for superficial carcinoma [3]. For example, intramucosal SCCs are curable using endoscopic resection, which avoids the morbidity and mortality associated with esophageal surgery. Thus, early screening of superficial SCC is crucial to improve prognosis. Patients with previous history of upper aerodigestive tract SCC (in particular ear, nose, and throat or esophageal SCC) have the highest risk of both metachronous and synchronous SCC in the whole upper aerodigestive tract [4-8].

In such patients, the European, American, and French gastrointestinal $(\mathrm{Gl})$ endoscopy societies recommend upper $\mathrm{Gl}$ endoscopy screening with esophageal chromoendoscopy $[4,9,10]$. Historically, the reference technique has been Lugol chromoendoscopy $[9,11-13]$. Although this technique is highly sensitive, its specificity is low, as demonstrated in several studies [14-16]. Furthermore, Lugol chromoendoscopy leads to esophageal spasms, is sometimes painful, and is time-consuming, resulting in infrequent use of Lugol staining by gastroenterologists for the screening of SCC (approximately $15 \%$ of screenings only) [17].

An alternative modality is narrow-band imaging (NBI), which is based on the use of selected wavelengths of light and has demonstrated high sensitivity and specificity to detect superficial SCC when performed by experts [14]. This technique is now recommended alongside Lugol chromoendoscopy for esophageal screening in European Society of Gastroenterology Endoscopy (ESGE) guidelines [10]. The sensitivity of NBI and Lugol chromoendoscopy is almost $100 \%$ in both modalities $[18,19]$, and the noninferiority (or even the superiority) of NBI over Lugol chromoendoscopy in terms of specificity has been reported but only in expert endoscopy centers. Nevertheless, to the best of our knowledge, only one study, reported by Ishihara et al. [20], has compared the diagnostic characteristics of NBI according to the level of expertise; the authors found that results from expert centers might not be reproducible in nonexpert settings.

The present study therefore aimed to compare the two techniques of chromoendoscopy in a nationwide randomized study involving expert and nonexpert centers to screen esophageal SCC in patients with a history or current SCC in the aerodigestive tract. The study aimed to demonstrate the superior specificity of NBI compared with Lugol chromoendoscopy to detect esophageal SCC and high grade dysplasia (HGD) in current practice (including tertiary care centers, local hospitals, and private clinics).

\section{Methods}

\section{Study design}

This was a multicenter, prospective, randomized, controlled, nonblinded study conducted in 15 French institutions comparing the detection of esophageal SCC using NBI vs. Lugol chromoendoscopy after a conventional white-light examination in both groups. As Lugol dyeing is still considered the gold standard for SCC detection, a Lugol examination was performed after the NBI examination in the NBI group to avoid any disadvantage to patients. Trial design is described in $>$ Fig. $\mathbf{1}$.

\section{Patients}

Patients scheduled for upper GI endoscopy were included if they had a history or current SCC and provided signed written consent to participate. Patients were excluded if they were aged less than 18 years, had a contraindication to upper $\mathrm{Gl}$ endoscopy or to general anesthesia, had a World Health Organization status 3 or 4 , were pregnant, or had not provided written informed consent.

Patients were recruited from 15 French institutions including four tertiary care centers and nine local hospitals or private clinics. As the aim was to evaluate real-life NBI specificity, NBI training was not provided as part of the study. Of note, Olympus high definition scopes with the NBI virtual chromoendoscopy function were already used by participating gastroenterologists in their current practice. 


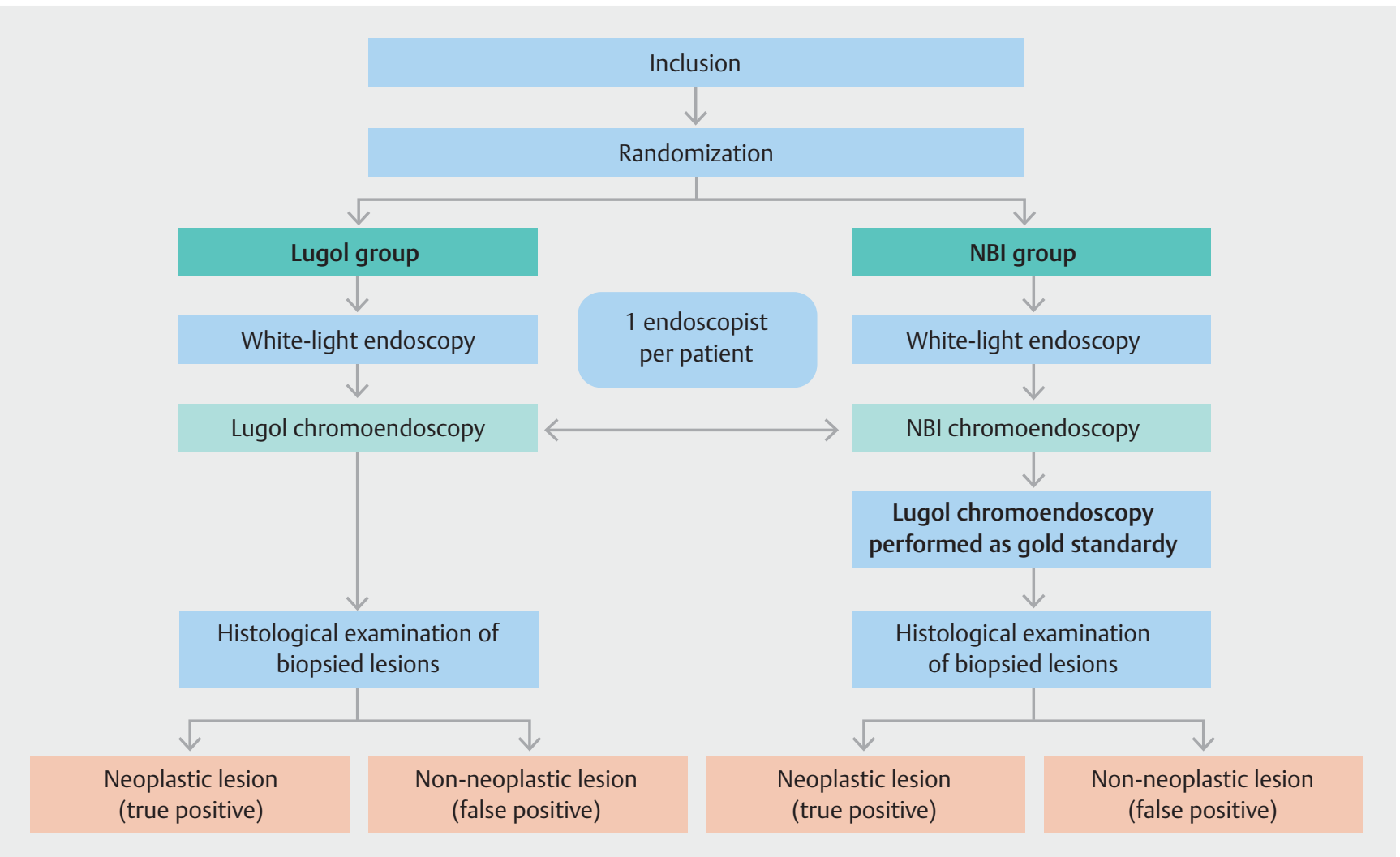

- Fig. 1 Trial design. NBI, narrow-band imaging.

\section{Interventions - diagnostic strategies}

All patients underwent an upper GI endoscopy, according to the protocols of each participating hospital in terms of patient position for anesthesia.

In both groups, an upper GI H180 series scope was used (Olympus, Tokyo, Japan). In cases of stenosis, an ultrathin GI transnasal scope (GIF N180; Olympus) could be used. The examinations were performed according to allocation.

In the Lugol group, two examinations were performed successively. First white-light imaging (WLI) examination was performed and all lesions detected were biopsied. Second, Lugol chromoendoscopy was performed (2.5\% Lugol dye was spread over the entire esophagus using a spray catheter, except near the upper esophageal sphincter owing to the risk of bronchospasm) and any additional lesions detected were reported separately from those detected by WLI and then biopsied for histological confirmation.

In the NBI group, three examinations were performed. First, WLI examination was performed and all lesions detected were described but not biopsied immediately to avoid bleedings that could alter the NBI examination. Second, NBI examination was performed and additional lesions detected were described separately. All lesions detected by both WLI and NBI were then biopsied at the same time, and the modality (WLI or NBI) that first detected each lesion was indicated on the report. Third, Lugol chromoendoscopy was performed; any additional lesions detected were reported separately from those detected by WLI/NBI and biopsied for pathological confirmation.

Diagnosis modality (WLI, NBI, or Lugol), size, topography, and macroscopic shape (Paris classification [21]) of each detected lesion were reported and described.

After the diagnostic procedure, no follow-up endoscopy was scheduled as part of this study.

\section{Outcomes}

The primary outcome was the specificity of the diagnostic strategies to detect high grade squamous cell neoplastic lesions (HGD and/or SCC) in patients with previous or current SCC in the aerodigestive tract in a per-patient analysis. The suspected lesions were defined by a pink color after Lugol staining [22], or by a color change or irregular vascular pattern using $\mathrm{NBI}$. All suspected lesions were biopsied for histological assessment to confirm or eliminate their neoplastic nature. If the lesion was confirmed to be neoplastic, it was a true positive for the detection modality, and if it was confirmed to be non-neoplastic it was defined as a false positive for the technique.

To evaluate the performance of each strategy, all lesions detected by each complete strategy (including in both cases a final chromoendoscopy with Lugol dye, the gold standard for detection, as presented in > Fig. 2), which were then confirmed by histological analysis of the biopsies taken, were used as reference. 


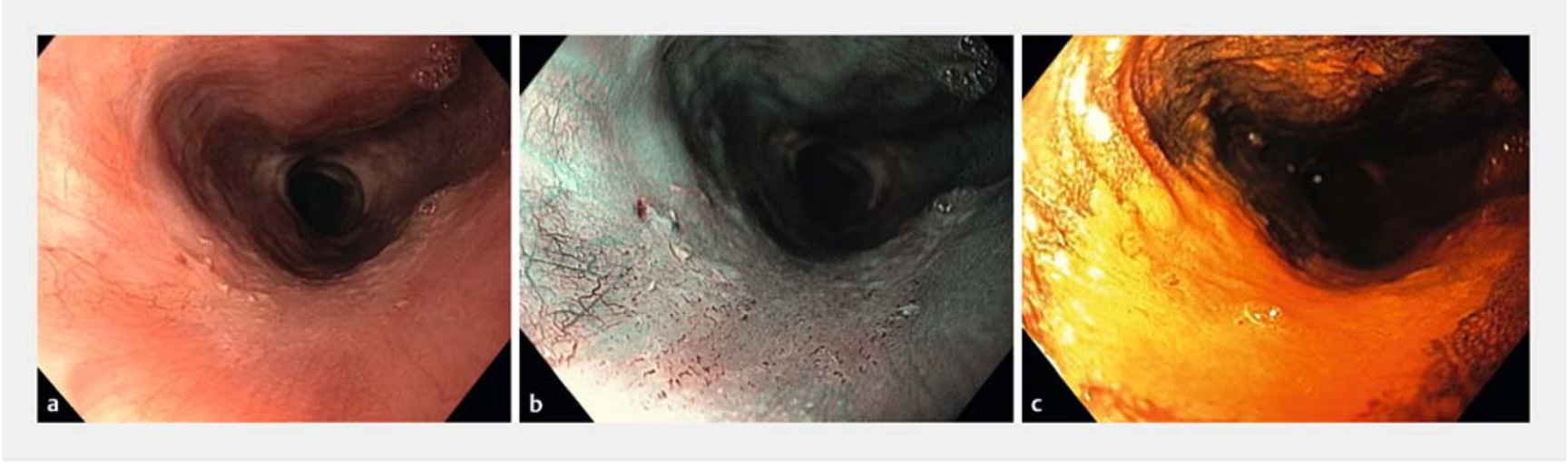

- Fig.2 A squamous cell carcinoma, as visualized by the three detection modalities. a White-light imaging. b Narrow-band imaging. c After Lugol's staining.

Secondary outcomes were sensitivity of the NBI strategy, positive predictive value (PPV), and negative predictive value (NPV) of the techniques in a per-patient analysis. A per-lesion analysis could be performed only for NBI as Lugol dyeing combined with histological assessment was considered the reference strategy for detection of esophageal SCC, with an assumed detection rate of $100 \%$.

\section{Sample size}

As previously reported, the sensitivity of NBI and Lugol chromoendoscopy are almost $100 \%[4,8,18,19]$. It was hypothesized that the per-patient specificity of NBI would be 12 percentage points greater than that of Lugol chromoendoscopy, which is assumed to be $80 \%[16,23]$. The sample size required was at least 320 patients for both groups using the Casagrande and Pike formula [24], for a 12 percentage point threshold of superiority and a statistical power of $80 \%$ with statistical significance defined as $P<0.05$ ( $\alpha=0.05$ and $\beta=0.20$ ), taking into account an exclusion or dropout rate of $10 \%$.

\section{Randomization}

Patients were randomly allocated in a 1:1 ratio to either the Lugol or NBI group. The randomization list was prepared by the independent clinical research associate of the French Society of Digestive Endoscopy (Société Française d'endoscopie digestive [SFED]) and stratified by center by random blocks of 4 to 6 . Sealed envelopes containing allocation arm were then sent to each center by the SFED clinical research associate. Patients were recruited by investigators who explained the study and collected the written consent at least 24 hours before the procedure. Each investigator opened the envelope immediately before the endoscopy procedure and the patient was blinded to the diagnostic strategy allocated.

\section{Histological evaluation}

All pathology samples were sent to expert digestive pathologists in each center and classified as follows: non-neoplastic tissue, low grade dysplasia (LGD), HGD, or SCC. Histological diagnoses were made according to the Vienna classification [25].

\section{Statistical methods}

Data were analyzed according to the intention-to-treat analysis principle with value set at zero (worst possible value) for patients with missing primary outcome data. Neoplastic lesion detection and miss rates were analyzed at the patient and lesion level.

Measures of diagnostic performance such as sensitivity, specificity, PPV, and NPV of the Lugol and NBI techniques to detect SCC and/or HGD were calculated in a per-patient analysis using histologically confirmed lesions identified by Lugol staining as the gold standard (Lugol staining was performed in both study arms). For per-patient analysis, detection of high grade neoplastic lesions was considered positive if at least one high grade neoplastic lesion was found by the diagnostic modality and confirmed histologically. A per-lesion analysis could be performed only for NBI as Lugol dyeing was considered the gold standard strategy for detection of SCC, with an assumed sensitivity for detection of $100 \%$. The $95 \%$ confidence intervals (Cls) were calculated for these estimates.

At the patient level, within-center comparisons between groups were performed using Mantel-Haenzel. At the lesion level, a generalized estimating equation approach was used adjusted for center. Sensitivity analysis included a per-protocol analysis to exclude patients with protocol deviations. Qualitative variables were compared using the Fisher's exact test. Quantitative variables were compared using Student's $t$ test. $P$ values of $<0.05$ were considered statistically significant. Statistical analyses were performed using SAS version 9.4 (SAS Institute, Inc., Cary, North Carolina, USA).

\section{Ethical concerns}

The study was performed in accordance with the Declaration of Helsinki and was approved by the regional ethics committee (Comité de Protection des Personnes Sud-Est III; number: 2010-045-B) and by the national medicine agency (Agence Francaise de Sécurité Sanitaire des Produits de Santé; number 2008-A01548-47). The study was registered at ClinicalTrials. gov (NCT04224896). 


\section{Results}

\section{Patients}

From March 2011 to December 2015, 335 patients with previous or current SCC were recruited; 334 were included (1 patient under tutorship was excluded due to erroneous inclusion), randomized to either the Lugol group $(n=167)$ or the NBI group $(n=167)$, and analyzed by intention-to-treat.

A total of 19 patients did not receive the allocated interventions including 8 in the Lugol group and 11 in the NBI group for the following reasons: 7 protocol deviations occurred during the endoscopy procedure (patients did not receive final Lugol chromoendoscopy), 6 withdrew their consent, 3 endoscopy procedures failed (orotracheal intubation failure, organizational issue, technical issue), 2 patients died before endoscopy, and 1 patient showed contraindication to general anesthesia before any procedure was performed ( $>$ Fig. 3 ).

\section{Baseline data and endoscopic procedure characteristics}

In both groups, most patients were male (Lugol group $91.0 \%$, NBI group $86.8 \%$ ), the mean age was 61 years, and most previous or current SCCs occurred in the ear-nose-throat tract (Lugol group $90.4 \%$, NBI group $80.8 \%$ ). The majority of patients smoked (Lugol group 89.2\%, NBI group 91.6\%) and/or consumed alcohol, either currently or in the past (Lugol group $85.6 \%, \mathrm{NBI}$ group $79.6 \%$ ) with a mean alcohol consumption of $68.1 \mathrm{~g}$ (standard deviation [SD] 61.4) and 69.3g (SD 78.9) in the Lugol and NBI groups, respectively (see Table $1 \mathrm{~s}$ in the online-only supplementary material).

According to the endoscopy procedure characteristics (Table $2 \mathbf{s}$ ), most patients in the two groups underwent a procedure under general anesthesia with or without tracheal intubation (Lugol group 67.1\%, NBI group 66.5\%). One patient had esophageal stenosis requiring the use of an ultrathin GI scope.

\section{Detection modality}

A total of 106 suspected lesions were detected in the Lugol group, 18 (17.0\%) of which (14 SCC, 1 HGD, 3 LGD) were confirmed histologically, in 16 patients ( $\downarrow$ Table 1 ). Among these 18 lesions, 11 lesions ( 10 SCC and 1 LGD) were detected during WLI examination. Among the 10 SCC detected with WLI, 1 was a T3, 1 was a T2, and 8 were T1 cancers. Lugol chromoendoscopy led to the detection of 7 additional superficial neoplastic lesions (4 T1 SCC, 1 HGD, 2 LGD) including 6 lesions (4 SCC, 1 HGD, 1 LGD) diagnosed in 6 additional patients in whom no synchronous lesion was detected by WLI.

In the NBI group, immediately after NBI examination, there were a total of 61 suspected lesions, 22 (36.1\%) of which (22 SCC) were confirmed histologically, in 18 patients. Among these 22 lesions, 19 T1 cancers and 2 T2 cancers were diagnosed by WLI examination. Additionally, one superficial T1 SCC was detected after NBI chromoendoscopy in one patient who did not have any synchronous lesion detected by WLI. There was no statistically significant difference in the number of patients with high grade neoplastic lesions detected between the Lugol and NBI groups ( $8.4 \%$ vs. $10.8 \% ; P=0.58)(\triangleright$ Table

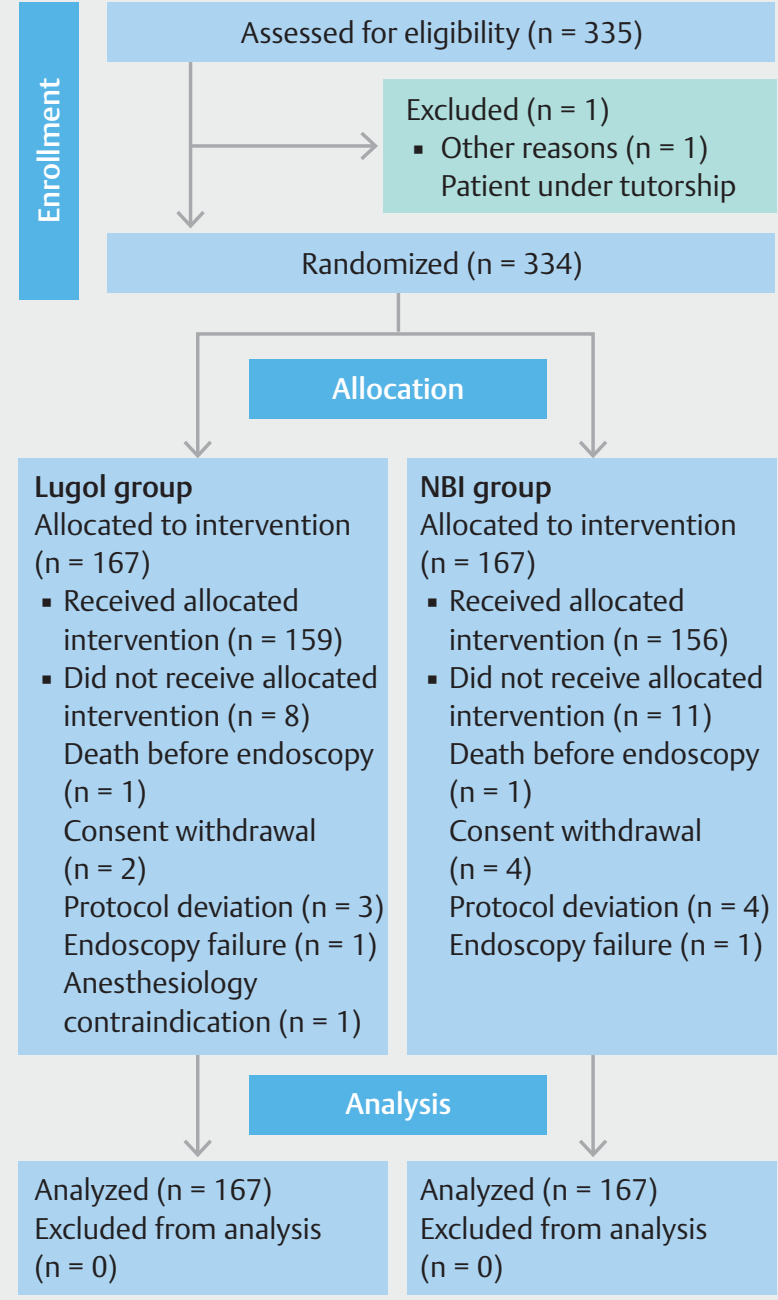

- Fig. 3 Flow chart. NBI, narrow-band imaging.

1). In the NBI group, Lugol chromoendoscopy detected 9 additional neoplastic lesions (2 T1 SCC, 2 HGD, 5 LGD) including 6 (2 SCC, 2 HGD, 2 LGD) in patients with synchronous SCC already detected with WLI or NBI, and 3 additional LGD that were diagnosed in 3 patients who had no high grade neoplastic lesion detected by NBI.

\section{Diagnostic performance of Lugol chromoendoscopy and NBI for HGD and SCC}

Per-patient analysis

For the detection of SCC and/or HGD, the sensitivity of Lugol was $100 \%$ (95\%Cl $76.8 \%-100 \%)$, specificity was $66.0 \%$ (95\% $\mathrm{Cl} 57.9 \%-73.5 \%)$, PPV was $21.2 \%$ (95\%Cl $12.1 \%-33.0 \%)$, and NPV was $100 \%(95 \% \mathrm{Cl} 96.4 \%-100 \%)$. The sensitivity of NBI was $100 \%$ (95\% Cl $81.5 \%-100 \%)$, specificity was $79.9 \%$ (95\% $\mathrm{Cl} 72.5 \%-86.0 \%)$, PPV was $37.5 \%$ (95\%Cl $24.0 \%-52.6 \%)$, and NPV was $100 \%(95 \% \mathrm{Cl} 96.9 \%-100 \%)$. The specificity of $\mathrm{NBI}$ was significantly greater than that of Lugol chromoendoscopy $(P=0.002)(\triangleright$ Table 2$)$. 
- Table 1 Characteristics of neoplastic lesions identified and confirmed histologically.

\begin{tabular}{|c|c|c|c|}
\hline & $\begin{array}{l}\text { Lugol } \\
n=167^{1}\end{array}$ & $\begin{array}{l}\text { NBI } \\
n=167^{1}\end{array}$ & $P$ value ${ }^{2}$ \\
\hline Patients with suspected lesions, $\mathrm{n}(\%)^{3}$ & $66(39.5)$ & $48(28.7)$ & 0.04 \\
\hline Suspected lesions (including non-neoplastic), $\mathrm{n}$ & 106 & 61 & \\
\hline - Total neoplastic lesions ${ }^{4}, \mathrm{n}(\%)$ & $18(17.0)$ & $22(36.1)$ & \\
\hline - SCC & 14 & 22 & \\
\hline . HGD & 1 & 0 & \\
\hline . LGD & 3 & 0 & \\
\hline \multicolumn{4}{|l|}{ Neoplastic lesions with WLI, n } \\
\hline - SCC & 10 & 21 & \\
\hline " HGD & 0 & 0 & \\
\hline . LGD & 1 & 0 & \\
\hline \multicolumn{4}{|l|}{ Additional neoplastic lesions detected after chromoendoscopy, n } \\
\hline - SCC & 4 & 1 & \\
\hline " HGD & 1 & 0 & \\
\hline . LGD & 2 & 0 & \\
\hline Patients with neoplastic lesion(s), n (\%) [95\%Cl] & $16(9.6)[5.6-15.1]$ & $18(10.8)[6.5-16.5]$ & 0.72 \\
\hline Patients with high-grade ${ }^{5}$ neoplastic lesion(s), n (\%) [95\%Cl] & $14(8.4)[4.7-13.7]$ & $18(10.8)[6.5-16.5]$ & 0.58 \\
\hline Neoplastic lesions per patient & & & 0.71 \\
\hline - Mean (SD) & $0.11(0.35)$ & $0.13(0.42)$ & \\
\hline - Range & $0-2$ & $0-3$ & \\
\hline High grade lesions per patient & & & 0.42 \\
\hline - mean (SD) & $0.09(0.31)$ & $0.16(0.53)$ & \\
\hline - Range & $0-2$ & $0-4$ & \\
\hline \multicolumn{4}{|c|}{$\begin{array}{l}\text { NBI, narrow-band imaging; SCC, squamous cell carcinoma; HGD, high grade dysplasia; LGD, low grade dysplasia; CI, confidence interval; SD, standard dev } \\
{ }^{1} \text { Patient number. } \\
{ }^{2} \text { Chi-squared test for qualitative variables and Wilcoxon's test for quantitative variables. } \\
{ }^{3} \text { Suspected lesions included neoplastic lesions and false positives (lesions detected but not confirmed to be neoplastic after histological examination). } \\
{ }^{4} \text { Confirmed neoplastic lesions after histological examination. } \\
{ }^{5} \text { High grade neoplastic lesions included HGD and SCC but excluded LGD. }\end{array}$} \\
\hline
\end{tabular}

Per-lesion analysis

For the detection of SCC and/or HGD lesions, the sensitivity of $\mathrm{NBI}$ was $84.0 \%(95 \% \mathrm{Cl} 63.9 \%-95.5 \%)$, specificity was $59.8 \%$ (95\% Cl 49.0\%-69.9\%), PPV was $93.2 \%$ (95\% Cl 83.5\%- $98.1 \%)$, and NPV was $36.2 \%(95 \% \mathrm{Cl} 24.0 \%-49.9 \%)$.

\section{Characteristics of the lesions detected}

The characteristics of the lesions detected in the two groups are presented in $>$ Table 3 . Across the entire patient cohort, there was no difference in size and extent of SCCs detected with WLI and Lugol chromoendoscopy, with mean length of $4.1 \mathrm{~cm}$ and $4.0 \mathrm{~cm}$, respectively $(P=0.93)$ and mean circumferential extension of $43.6 \%$ and $37.5 \%$, respectively $(P=0.64)$. The only SCC detected with NBI measured $1 \mathrm{~cm}$ and was estimated to extend to $8.3 \%$ of the circumference.

\section{Adverse events}

One patient from the Lugol group experienced severe respiratory distress during anesthesia induction, prior to any endoscopic examination ( $0.6 \%$ morbidity).

\section{Per-protocol analyses}

Results of the per-protocol analyses were similar to those of the intention-to-treat analyses (results not presented).

\section{Discussion}

The present study confirms previously published data from expert endoscopists reporting that NBI is more specific than Lugol for the diagnosis of SCC and/or HGD $[14,15]$. Sensitivity of NBI was $100 \%$ in a per-patient analysis although per-lesion evalua- 
- Table 2 Diagnostic performance of Lugol and narrow-band imaging for detection of squamous cell carcinoma and high grade dysplasia (per-patient analysis).

\begin{tabular}{|c|c|c|c|}
\hline & $\begin{array}{l}\text { Lugol group } \\
n=167\end{array}$ & $\begin{array}{l}\text { NBI group } \\
n=167\end{array}$ & $P$ value* \\
\hline Sensitivity, \% (95\%Cl) & $100(76.8-100)$ & $100(81.5-100)$ & NC \\
\hline Specificity, \% (95\%Cl) & $66.0(57.9-73.5)$ & $79.9(72.5-86.0)$ & 0.002 \\
\hline PPV, \% (95\%CI) & $21.2(12.1-33.0)$ & $37.5(24.0-52.6)$ & 0.001 \\
\hline NPV, \% (95\%CI) & $100(96.4-100)$ & $100(96.9-100)$ & NC \\
\hline
\end{tabular}

tion showed only $84 \%$ sensitivity with no HGD or LGD lesions detected. These results are in line with those reported by Nagami et al. who compared the two modalities performed by expert endoscopists, and found that the specificity of NBI (79.2\%) was significantly greater than that of Lugol chromoendoscopy (64.0\%; $P=0.01$ ) but that there was no statistically significant difference in sensitivity ( $88.3 \%$ vs. $94 \% ; P=0.67)$ [15]. In a recent meta-analysis including 1123 patients, Morita et al. also reported no difference between NBI and Lugol chromoendoscopy regarding sensitivity (per-patient analysis $88 \%$ vs. $92 \%$ ) but a statistically significant higher specificity for NBI vs. Lugol (per-patient analysis $88 \%$ vs. $82 \%$ ) [14].

Thus, NBI results obtained by experts appear reproducible in general gastroenterology practice and NBI should thus become the reference method for esophageal cancer screening, as it is currently far easier to use than Lugol. More specifically, many side effects of Lugol chromoendoscopy are known (i.e. esophagitis, gastritis, chest pain, esophagospasm, bronchospasm) [4], and the difficulties associated with its application are likely to explain its low use (approximatively $15 \%$ of SCC screenings in France [17]). Furthermore, the upper part of the esophagus and pharyngeal areas are frequently not colored by Lugol, reducing the detection of synchronous or metachronous neoplastic lesions in these locations.

Magnification is not currently available in most nonexpert settings in France and was not used in this study, where 180 series endoscopes were used. Nevertheless, this study confirms that the specificity of nonmagnified $\mathrm{NBI}$ is still higher than that of Lugol chromoendoscopy. Thus, NBI with magnification, already more specific than nonmagnified NBI could be associated with even better results and should be implemented in our general practice. In addition, the specificity of Lugol staining is low, despite the use of the pink color sign to define the suspected lesions, demonstrating the subjective character of such a color sign, particularly when involving nonexpert endoscopists who do not frequently see squamous neoplastic lesions.

Nevertheless, we report a lower sensitivity of NBI examination in a per-lesion analysis, especially for LGD and HGD (NBI group LGD $n=0 / 5$ and HGD $n=0 / 2$ ) compared with Lugol chromoendoscopy (NBI group LGD $n=5 / 5$ and HGD $n=2 / 2$ ). This low sensitivity is probably related to the low level of endoscopist experience with NBI in this real-life study. Ishihara et al.
[20] reported a lower sensitivity in the detection of esophageal HGD when NBI was performed by nonexperts (53\%) compared with experts ( $100 \%$; $P<0.001)$. Moreover, a learning curve was also described in the study by Ishihara et al., showing great improvement in HGD detection according to increased NBI utilization. In certain studies [15, 26], a teaching course was provided before conducting an NBI-related study to improve experience in identifying vascular and mucosal abnormalities [27]. In the present study, the aim was to evaluate NBI performance in routine conditions without prior teaching courses that could artificially improve results.

Furthermore, NBI improves detection in the pharyngeal area and the upper esophagus without the morbidity associated with Lugol, including aspiration, pain, or esophageal spasms [28]. Recent ESGE guidelines recommend NBI as an alternative to Lugol chromoendoscopy in esophageal SCC detection [10, 29]. Nevertheless, nine neoplastic lesions were detected by Lugol dye after they had been missed with NBI, demonstrating that Lugol dyeing, although not very easy to use as a screening strategy, could improve the detection of synchronous SCC particularly when a lesion has already been detected by NBI. The combination of the two techniques could probably improve the results of detection when NBI is used as the initial screening strategy.

The present study has several limitations. First, a single endoscopist performed the different examinations without crossover, precluding blind evaluation of each diagnostic modality. Furthermore, owing to the design of the study, Lugol chromoendoscopy sensitivity was not measurable and was thus considered to be $100 \%$, and Lugol chromoendoscopy specificity calculation was only possible in per-patient analysis and not in a per-lesion analysis. Magnification was not used as this technique was not in widespread use in France during the study period. Finally, endoscopist skills were not evaluated and therefore diagnostic performance of NBI and Lugol chromoendoscopy could not be analyzed according to physicians' expertise.

In conclusion, this study confirms previous studies conducted in expert centers, or at least by expert endoscopists, showing that NBI could replace Lugol chromoendoscopy to detect SCC and HGD in the esophagus, even in general gastroenterology practice, possibly changing current recommendations. However, when an SCC is detected by a nonexpert during NBI 
Table 3 Endoscopic description of the lesions detected in the two groups.

\begin{tabular}{|c|c|c|c|}
\hline & Lugol group & NBI group & P value* \\
\hline Total neoplastic lesions, $\mathrm{n}(\%)$ & 18 & 31 & \\
\hline . SCC & 14 & 24 & \\
\hline . HGD & 1 & 2 & \\
\hline . LGD & 3 & 5 & \\
\hline Paris classification description of SCCs ${ }^{1}$ & & & 0.90 \\
\hline - Type I & 5 & 5 & \\
\hline - Type lla & 2 & 8 & \\
\hline - Type IIb & 6 & 6 & \\
\hline - Type Ilc & 1 & 2 & \\
\hline " Type III & 0 & 1 & \\
\hline - Missing data & 0 & 2 & \\
\hline Mean length of neoplastic lesions ( $\mathrm{n}$ ) detected with WLI, cm & $3.4(11)$ & $4.3(21)$ & 0.30 \\
\hline . SCC & $3.6(10)$ & $4.3(21)$ & 0.45 \\
\hline - HGD & $\mathrm{NA}(0)$ & $\mathrm{NA}(0)$ & \\
\hline - LGD & $1.0(1)$ & $\mathrm{NA}(0)$ & \\
\hline Mean length of additional neoplastic lesions ( $\mathrm{n}$ ) detected with NBI, cm & NA & $1.0(1)$ & NC \\
\hline . SCC & NA & $1.0(1)$ & \\
\hline . HGD & NA & $\mathrm{NA}(0)$ & \\
\hline . LGD & NA & $N A(0)$ & \\
\hline Mean length of additional neoplastic lesions ( $\mathrm{n}$ ) detected with Lugol, $\mathrm{cm}$ & $3.4(7)$ & $2.4(9)$ & 0.43 \\
\hline . SCC & $5.3(4)$ & $1.5(2)$ & 0.14 \\
\hline . HGD & $1.0(1)$ & $4.5(2)$ & NC \\
\hline . LGD & $1.0(2)$ & $3.8(5)$ & 0.24 \\
\hline Mean circumferential extension of lesions (n) detected with WLI, \% & $37.5(11)$ & $44.8(21)$ & 0.49 \\
\hline . SCC & $40.8(10)$ & $44.8(21)$ & 0.72 \\
\hline . HGD & $\mathrm{NA}(0)$ & $\mathrm{NA}(0)$ & \\
\hline$\cdot$ LGD & $1.0(1)$ & $\mathrm{NA}(0)$ & \\
\hline Mean circumferential extension of lesions (n) detected with NBI, \% & NA & $8.3(1)$ & NC \\
\hline . SCC & NA & $8.3(1)$ & NC \\
\hline - HGD & NA & $\mathrm{NA}(0)$ & \\
\hline . LGD & NA & $N A(0)$ & \\
\hline Mean circumferential extension of lesions ( $\mathrm{n}$ ) detected with Lugol, \% & $34.7\left(6^{1}\right)$ & $45.3(9)$ & 0.97 \\
\hline . SCC & $47.9(4)$ & $16.7(2)$ & 0.33 \\
\hline - HGD & $8.3(1)$ & $66.6(2)$ & NC \\
\hline . LGD & $8.3\left(1^{1}\right)$ & $33.4(5)$ & NC \\
\hline
\end{tabular}

NBI, narrow-band imaging; SCC, squamous cell carcinoma; HCD, high grade dysplasia; LGD low grade dysplasia; WLI, white-light imaging; NA, not applicable; NC, not calculable.

* One additional missing data. 
examination, an additional Lugol examination of the whole esophagus could be proposed to improve detection of synchronous dysplastic lesions, which are easily missed by nonexperts.

\section{Acknowledgment}

This study was supported by SFED.

\section{Clinical trial}

Trial Registration: ClinicalTrials.gov | Registration number (trial ID): NCT04224896 | Type of study: randomized controlled study.

Competing interests

The authors declare that they have no conflicts of interest.

References

[1] Anderson LA, Tavilla A, Brenner $\mathrm{H}$ et al. Survival for oesophageal, stomach and small intestine cancers in Europe 1999-2007: results from EUROCARE-5. Eur J Cancer 2015; 51: 2144-2157

[2] Torre LA, Siegel RL, Ward EM et al. Global cancer incidence and mortality rates and trends - an update. Cancer Epidemiol Prev Biomark 2016; 25: 16-27

[3] Zhang Y. Epidemiology of esophageal cancer. World J Gastroenterol 2013; 19: 5598-5606

[4] Dubuc J, Legoux J-L, Winnock M et al. Endoscopic screening for esophageal squamous-cell carcinoma in high-risk patients: a prospective study conducted in 62 French endoscopy centers. Endoscopy 2006; 38: 690-695

[5] Ina $\mathrm{H}$, Shibuya $\mathrm{H}$, Ohashi I et al. The frequency of a concomitant early esophageal cancer in male patients with oral and oropharyngeal cancer. Screening results using Lugol dye endoscopy. Cancer 1994; 73: 2038-2041

[6] Ribeiro U, Posner MC, Safatle-Ribeiro AV et al. Risk factors for squamous cell carcinoma of the oesophagus. Br J Surg 1996; 83: 11741185

[7] Petit T, Georges C, Jung GM et al. Systematic esophageal endoscopy screening in patients previously treated for head and neck squamouscell carcinoma. Ann Oncol 2001; 12: 643-646

[8] Muto M, Hironaka S, Nakane M et al. Association of multiple Lugolvoiding lesions with synchronous and metachronous esophageal squamous cell carcinoma in patients with head and neck cancer. Gastrointest Endosc 2002; 56: 517-521

[9] Hirota WK, Zuckerman M], Adler DG et al. Standards of Practice Committee, American Society for Gastrointestinal Endoscopy. ASGE guideline: the role of endoscopy in the surveillance of premalignant conditions of the upper GI tract. Gastrointest Endosc 2006; 63: 570580

[10] Pimentel-Nunes P, Dinis-Ribeiro M, Ponchon T et al. Endoscopic submucosal dissection: European Society of Gastrointestinal Endoscopy (ESGE) Guideline. Endoscopy 2015; 47: 829-854

[11] Bisschops R, Areia M, Coron E et al. Performance measures for upper gastrointestinal endoscopy: a European Society of Gastrointestinal Endoscopy (ESGE) Quality Improvement Initiative. Endoscopy 2016; 48: $843-864$
[12] Kandiah K, Chedgy FJQ, Subramaniam S et al. Early squamous neoplasia of the esophagus: the endoscopic approach to diagnosis and management. Saudi J Gastroenterol 2017; 23: 75-81

[13] Trivedi PJ, Braden B. Indications, stains and techniques in chromoendoscopy. QJM 2013; 106: 117-131

[14] Morita FHA, Bernardo WM, Ide E et al. Narrow band imaging versus Lugol chromoendoscopy to diagnose squamous cell carcinoma of the esophagus: a systematic review and meta-analysis. BMC Cancer 2017; 17: 54

[15] Nagami $\mathrm{Y}$, Tominaga $\mathrm{K}$, Machida $\mathrm{H}$ et al. Usefulness of non-magnifying narrow-band imaging in screening of early esophageal squamous cell carcinoma: a prospective comparative study using propensity score matching. Am J Gastroenterol 2014; 109: 845-854

[16] Ide E, Maluf-Filho F, Chaves DM et al. Narrow-band imaging without magnification for detecting early esophageal squamous cell carcinoma. World J Gastroenterol 2011; 17: 4408-4413

[17] Canard JM, Arpurt JP, Boustière $C$ et al. la Lettre de la SFED. Deux jours d'endoscopie en France. Résultats de l'enquête 2006. 2007: http:// www.sfed.org/files/documents_sfed/files/lettresfed/Lettre_SFED_ No36.pdf

[18] Ponchon T, Lapalus MG, Saurin JC et al. Could narrow band imaging (NBI) replace Lugol staining for the detection of esophageal squamous cell carcinoma? Gastrointest Endosc 2007; 65: AB343

[19] Tincani AJ, Brandalise N, Altemani A et al. Diagnosis of superficial esophageal cancer and dysplasia using endoscopic screening with a $2 \%$ Lugol dye solution in patients with head and neck cancer. Head Neck 2000; 22: 170-174

[20] Ishihara R, Takeuchi Y, Chatani R et al. Prospective evaluation of narrow-band imaging endoscopy for screening of esophageal squamous mucosal high-grade neoplasia in experienced and less experienced endoscopists. Dis Esophagus 2010; 23: 480-486

[21] The Paris endoscopic classification of superficial neoplastic lesions: esophagus, stomach, and colon: November 30 to December 1, 2002. Gastrointest Endosc 2003; 58: S3-43

[22] Shimizu Y, Omori T, Yokoyama A et al. Endoscopic diagnosis of early squamous neoplasia of the esophagus with iodine staining: highgrade intra-epithelial neoplasia turns pink within a few minutes. J Gastroenterol Hepatol 2008; 23: 546-550

[23] Takenaka R, Kawahara Y, Okada $\mathrm{H}$ et al. Narrow-band imaging provides reliable screening for esophageal malignancy in patients with head and neck cancers. Am J Gastroenterol 2009; 104: 2942-2948

[24] Casagrande JT, Pike MC. An improved approximate formula for calculating sample sizes for comparing two binomial distributions. Biometrics 1978; 34: 483-486

[25] Dixon MF. Gastrointestinal epithelial neoplasia: Vienna revisited. Gut 2002; 51: 130-131

[26] Muto M, Minashi K, Yano T et al. Early detection of superficial squamous cell carcinoma in the head and neck region and esophagus by narrow band imaging: a multicenter randomized controlled trial. J Clin Oncol 2010; 28: 1566-1572

[27] Inoue H, Kaga M, Ikeda $\mathrm{H}$ et al. Magnification endoscopy in esophageal squamous cell carcinoma: a review of the intrapapillary capillary loop classification. Ann Gastroenterol 2015; 28: 41-48

[28] Jin D, Wang J, Zhan Q et al. The safety and efficacy of $2 \%$ vitamin C solution spray for relief of mucosal irritation caused by Lugol chromoendoscopy: a multicenter, randomized, double-blind, parallel trial. Gastrointest Endosc 2019: doi:10.1016/j.gie.2019.11.028

[29] Bories E, Barret M, Chaussade S. Endoscopic treatment of superficial oesophageal squamous cell cancers. Acta Endosc 2017; 47: 192-194 